Infusionstherapie 1986;13:297-298

\title{
Jahres-Inhalt 1986
}

Abstrakts zur 5. Gemeinsamen Jahrestagung der Österreichischen und Deutschen Arbeitsgemeinschaften für künstliche Ernährung (AKE/DAKE), 13.-15. 3. 1986, Salzburg 4 McCartney, S. F.iAppel, P. L.; Shoemaker, W. C. und Beez, M. G., Los Angeles, Calif./USA Die Reaktionen kritisch kranker Patienten unter Volu-mentherapie mit Hydroxyäthylstärke (6 \% HES 450/0,7) 34

Buchbesprechungen 41

Informationen für die Klinik 42

Margot Semsroth, WienlÖsterreich

Indirekte Kalorimetrie bei beatmeten Kindern - 3.Teil:

Klinische Anwendung eines neuen Meßverfahrens .... 44

Daly, J. M., New York/USA

Malnutrition und metabolische Mangelerscheinungen bei

Krebspatienten* 66

Leserzuschrift 69

Ollenschläger, G., Köln

Einfluß aggressiver Tumortherapie auf die Nährstoffver-

wertung und den Ernährungszustand 70

Sonderbände 77

Lehnert, H., Münster

Merkmale und Mechanismen der Tumoranorexie .... 78

Buchbesprechung 84

Eígenbrodt, E. und Reinacher, M., Gießen Kohlenhydratstoffwechsel in neoplastischen Geweben .85

Informationen für die Klinik 91

Striebel, J.-P.; Saeger, H.-D.; Ritz, R.; Leweling, H. und Holm, E., Mannheim

Amitosäurenaufnahme und -abgabe kolorektaler Karzi-

nome des Menschen 92

Erratum 105

Nachtrag Abstrakt Krawczyk et al. , Warschau

zur 5. Jahrestagung der AKE/DAKE, März 1986, Salz

burg 105

Ruffmann, R.; Tartaris, T. und Chirigos, M. A.,

Frederick, Md.IUSA und Schlick, E., Ludwigshafen

Proteinmangel, Körperabwehr und natürliche Anti-

Tumor-Immunität 106

M. E. Heim, Mannheim

Adjuvante parenterale Ernährung bei zytostatischer 
Chemotherapie

115

Grünert, A. und Heim, F., Ulm

Adjuvante parenterale Ernährung bei chemo- und radio-

therapeutischen Maßnahmen in der Hämatologie .... 122

Mutter, J. M.; Keller, H. W.; Brenner, U.; Walter, M. und

Holzmüller, W., Köln-Lindenthal

Adjuvante künstliche Ernährung in der Tumorchirurgie 126

Keymling, M. und Warner, W., Bad Hersfeld

Möglichkeiten für den Einsatz von Formuladiäten bei

Tumorpatienten $\quad 134$

H. Rasper, Würzburg

Gibt es eine diätetische Therapie maligner Tumoren? . 142

Informationen für die Klinik 144

Boldt, J.; v. Bormann, B.; Kling, D.; Börner, U.;

Mulch, J. und Hempelmann, G., Gießen

Volumenersatz mit einem neuen Hydroxyäthylstärke-

Präparat (3\% HAS 200/0,5) in der Herzchirurgie 145

Pfenninger, E.; Grünert, A.; John, U. und Wiedeck, H.,

Ulm

Klinische Untersuchung zur bakteriellen Kontamination

zweierverschieden zubereiteter enter alerNährlösungen . 152

Buchbesprechungen 158

Vorwort 161

Wernerman, J.; von der Decken, A. und Vinnars, E., Stockholm/Schweden

Die Interpretation von Ribosomenbestimmungen für die

Beurteilung der Proteinsynthese im menschlichen Ske-

lettmuskel 162

Guarnieri, G.; Toigo, G.; Situlin, R.; Crapesi, L.;

Del Bianco, M.A.; Zanettovich, A.; Mandero, E.

und Resetta, G. , Triestlltalien

Untersuchungen an Muskel-Biopsieproben über Protein-

Energie-Mangelernährung bei Patienten mit chronisch-

rezidivierender Pankreatitis 166

Roth, E.; Zöch, G.; Mauritz, W.; Karner, J.;

Funovícs, J.; Sporn, P. und Fritsch, A., Wienl Österreich

Stoffwechselveränderungen bei Patienten mit akuter

nekrotísierender Pankreatitis 172

$2 \% \mathrm{~S}$

Jahres-Inhalt 1986

Carpentier, Y.A. und Bihain, B.E., BrüssellBelgien,

Deckelbaum, R.J., New York/USA

Fettemulsionen sind mehr als Energielieferanten

Soeters, P.B. und van Leeuwen, P.A.M.,

Maastricht! Niederlande

Ammoniak- und Glutaminstoffwechsel des Darms. - Ein- 
fluß von Laktulose und Neomyzin . 186

Informationen für die Klinik 192

Vazquez, J.A.; Paleos, G.A.; Lochs, H.

und Adibi, S.A., Pittsburgh/USA,

Langer, K.; Brandl, M., Erlangen

Eine konzentrierte Mischung von Aminosäuren und

Dipeptiden für totale parenterale Ernährung 193

Buchbesprechungen 199

Balogh, D. und Hackl, J. M., Innsbruck, Legenstein, E., Wien, Musil, H. E., Graz (Österreich)

Erfahrungen mit L-Carnitin in der Postaggressions-

phase 204

Buchbesprechungen 1208

Sitges-Serra, A.; Figueras-Felip, J.; Alastrue-Vidal, A.;

Jaurrieta-Mas, E. und Rafecas-Renau, A.,

Barcelona (Spanien)

Randomisierte Studie zur Wirkung von isotonischen

Aminosäurenlösungen auf postoperative Komplikationen

und die Konzentration von Plasmaproteinen mit kurzer

Halbwertszeit 210

Nagler, J., Göppingen

Intraoperative Infusionstherapie. - Auswertung der

Zufuhrraten und Prüfung der Effektivität von Infusions-

wärmern 216

Finsterer, U. und Rötzer, R., München

Renale Effekte von Dopamin bei gesunden Erwachsenen unter besonderer Berücksichtigung der Exkretion von

Phosphat und Kalzium 222

Brenner, U.; Midler, J. M.; Walter, M.; Holzmüller, W. und Keller, H. W., Köln

Anthropometrische Parameter

Sonderbände 237

Informationen für die Klinik 238

Glück, D.; Kubanek, B. und Ahnefeld, F. W., Ulm

Die Therapie mit Blutkomponenten. - Voraussetzungen,

Indikationen und klinische Anwendung 240

Buchbesprechungen II 250

Bock, E., Nürnberg

Aminosäurenzufuhr beim akuten, noch kompensierten

Nierenversagen in der posttraumatischen Phase

Druml, W.; Kleinberger, G.; Balcke, P.;

Laggner, A.; Lenz, K.; Schmidt, P. und

Zazgornik, J., Wien/Österreich, Burger, U., Gießen

Elimination von Aminosäuren bei chronischem Nieren

versagen 262

Heller, W.; Musil, H. E.; Gaebel, G.; Hempel, V.; 
Krug, W. und Köhn, H. J., Tubingen

Der Einfluß von L-Carnítin auf den Postaggressionsstoffwechsel operierter Patienten 268

Matkowitz, R.; Junghans, P.; Hartig, W.; Jung, K.;

Faust, H.; Pester, A. und Ritter, R., Leipzig/DDR

Intravenöser $15 \mathrm{~N}-$ Verwertungstest zur Erfassung des Einflusses parenteraler Nahrungszufuhr auf die Proteinsynthese $\quad 277$

Informationen für die Klinik 282

Buchbesprechungen 285

Wicklmayr, M.; Rett, K.; Dietze, G. und Mehnert, H., München

Vergleichende Untersuchungen zur Metabolisierung von

MCT/LCT-und LCT-Emulsionen bei Diabetikern .... 287

Neuhäuser, M. und Bässler, K. H., Mainz

Die biologische Verfügbarkeit von Glutamin aus

N-Acetyl-L-Glutamin bei intravenöser Verabreichung.

- Untersuchungen an der Ratte 292

Jahres-Inhalt $1986 \quad 297$

Autorenverzeichnis $1986 \quad 299$

Supplement 1/August ' 86

Abstrakts zum 21. Kongreß der Deutschen Gesellschaft

für Bluttransfusion und Immunhämatologie

Hannover, 24.-27. September 1986 S1/3-36 